\title{
Sample Preparation of Epitaxial InN Layers for Transmission Electron Microscopy.
}

\author{
Z. Liliental-Weber ${ }^{*}$, M. E. Hawkridge ${ }^{*}$, D. Erwin ${ }^{* *}$ and M. Libbee ${ }^{* *}$ \\ *Materials Science Division, Lawrence Berkeley National Laboratory, 1 Cyclotron Road MS \\ 62R0213, Berkeley, CA 94720 \\ ** Gatan Inc., 5794 W. Las Positas Blvd., Pleasanton, CA 94588
}

Motivated in part by the exploitation of a band gap range covering the entire visible solar spectrum, research into $\mathrm{InN}$ and ternary InGaN alloys has seen rapid expansion in recent years. Preparation of high quality electron transparent samples is of critical importance for transmission electron microscopy (TEM) studies of these materials. In most cases, conventional mechanical thinning procedures are used with a final step of ion milling to produce thin specimens. However, the ion milling characteristics of InN are not terribly well defined; ion milling parameters are often inferred from other materials and assumed to be sufficient for the intended studies when this may not be the case. For example, preferential milling of phosphor has been observed in InP [1] and surface enrichment of arsenic occurs in InAs due to radiation-induced diffusion and segregation [2]. Pearton et al [3] did not observe preferential milling of polycrystalline InN during Ar ion bombardment up to $500 \mathrm{eV}$, but many conventional ion-milling machines are not capable of useful operation at such low energies. Here, we report on our attempts to prepare InN layers suitable for TEM analysis. We find that careful thermal management of the sample and use of low voltages are necessary during ion milling to prevent the formation of indium droplets and other artifacts detrimental for TEM analysis.

Fig. 1(a) shows a TEM image of a thick InN layer grown on a GaN template/ sapphire substrate prepared in a Gatan precision ion polishing system (PIPS) without a cold stage. For most materials, the thermal conductivity of the duopost stage is sufficient to prevent excess heating of the sample during ion milling. However, in this case the thick InN layer exhibits large droplets on the surface that limit the usable areas for TEM analysis. This is of particular importance when studying extended defects in thicker epitaxial layers and their relationship to opto-electronic properties. To suppress the formation of these artifacts, we cooled the samples during ion milling using the same model PIPS with a cold stage installed. Fig. 1(b) shows a TEM sample prepared from the same thick InN material using the same milling parameters as before. In this case, the large droplets are no longer observed. However, in some samples prepared using similar parameters, droplets were again observed due to the thermal conductivity of the samples being compromised. We discuss how this occurred and how in-situ monitoring of the samples by optical microscopy during ion milling can avoid such events from occurring.

For high-resolution experiments, a low-voltage cleaning step is usually used to remove any ion damage in the surface of the foil, including the oxide layer that causes the InN layer to appear muggy in Fig 1(b). Fig. 2 shows a TEM sample of a thick InN layer milled using the same parameters as previously plus LN cooling, but with a final cleaning step of $800 \mathrm{eV}$ (a typical cleaning energy for most materials) using a LoEnergy PIPS. In this case, the oxide layer appears to have been removed, but small dark spots are present on the InN layer, suggesting an accumulation of higher $\mathrm{Z}$ material. We believe that these spots are In-rich areas produced by the preferential sputtering of nitrogen from the surface of the foil at the lower ion beam energy as they are not observed elsewhere 
on the thin foil. We discuss the impact of these results when interpreting TEM measurements. Lastly, we show that ion milling at extremely low voltages for very brief periods are sufficient to avoid preferential sputtering while removing the surface damage layer.

References:

[1] J. B. Malherbe and W. O. Barnard, Surf. Sci. 255, 309 (1991)

[2] M. Tanemura, M. Ukita, and F. Okuyama, Surf. Sci. 426, 141 (1999)

[3] S. J. Pearton, C. R. Abernathy, F. Ren, and J. R. Lothian, J. Appl. Phys. 76, 1210 (1994)

[4] This work was supported by the U.S. Dept. of Energy under Contr. No. DE-AC02-05CH11231.
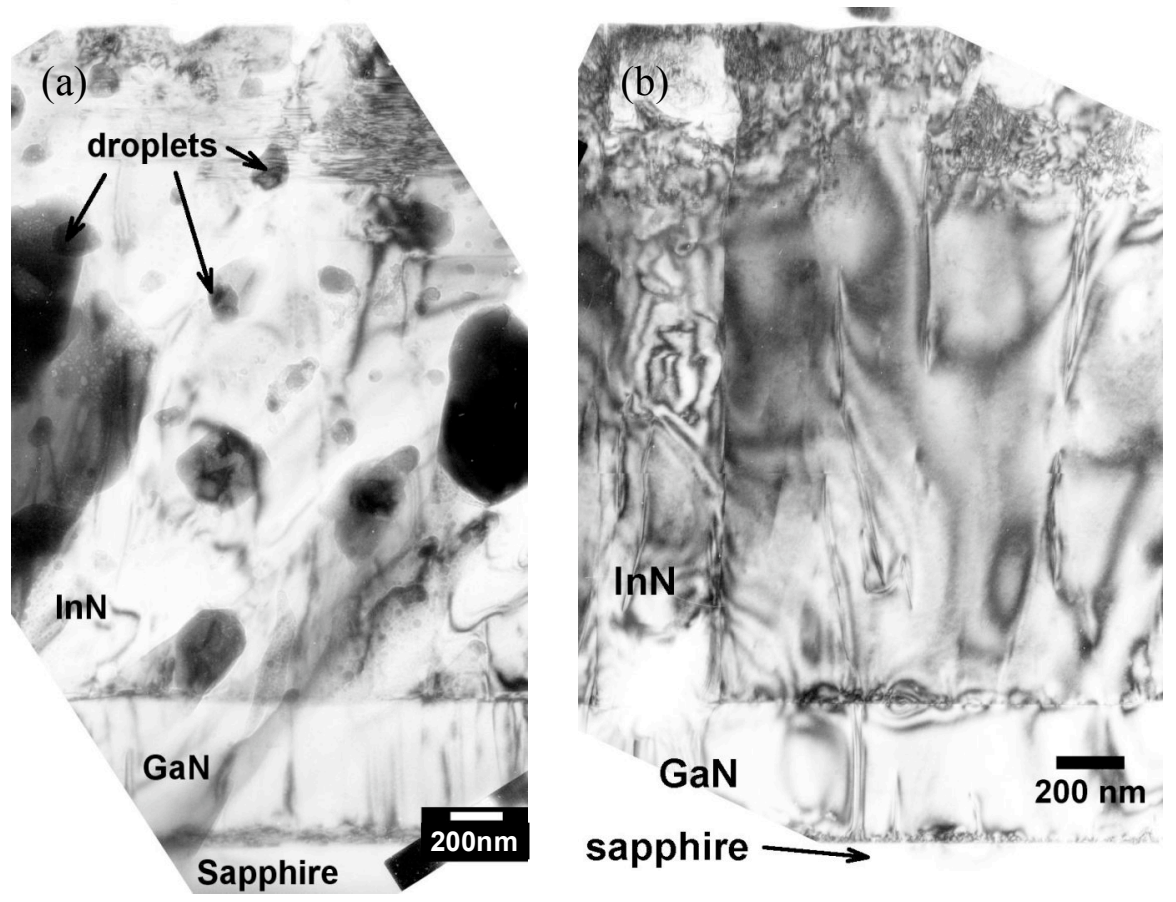

Fig. 1: (a) Bright field image of sample contaminated with large droplets that obstruct extended defect details; (b) Sample milled under identical conditions using cold stage (no droplets observed).

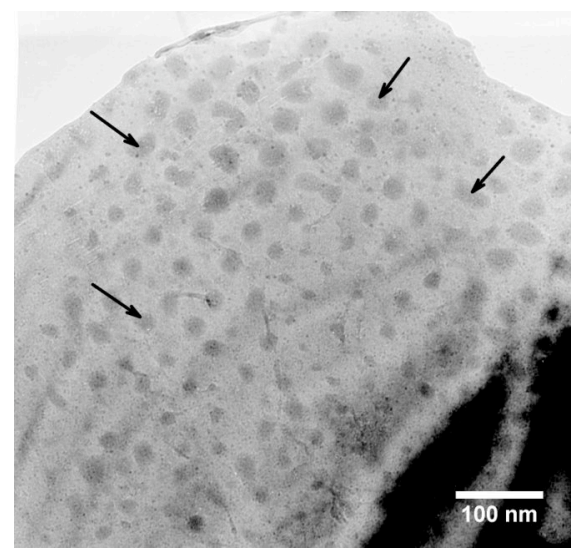

Fig. 2: Bright field TEM image of $800 \mathrm{eV}$ cleaned sample showing small dark spots on InN layer (examples arrowed), indicating higher $\mathrm{Z}$ areas. 